\title{
In situ FIB-SEM Experimentation: from Nanoscale Wetting to Nano- fabrication of Gallium-based Liquid Metals
}

\author{
K. Doudrick, ${ }^{1,2}$ S. Liu, ${ }^{1}$ E.M. Mutunga, ${ }^{3}$ K.L. Klein, ${ }^{3,4}$ K.K. Varanasi, ${ }^{5}$ and K. Rykaczewski ${ }^{*}$
}

1. School for Engineering of Transport, Matter and Energy, Arizona State University, Tempe, AZ 85287

2. School of Sustainable Engineering and The Built Environment, Arizona State University Tempe, AZ 85287

3. University of the District of Columbia, Washington, DC

4. Material Measurement Laboratory, National Institute of Standards and Technology, Gaithersburg, MD 20899

5. Department of Mechanical Engineering, Massachusetts Institute of Technology, Cambridge, MA 02139

Stretchable conductors are of interest for a number of applications including soft robotics, flexible electronics, and biomedical devices. ${ }^{[1]}$ The use of liquid metals is particularly attractive because, in contrast to semiconductor based stretchable electronics, ${ }^{[1]}$ circuitry based on these materials is intrinsically soft and remains functional even when stretched to several times its initial length. ${ }^{[2]}$ The first example of liquid-phase electronics is the Whitney strain gauge, ${ }^{[3]}$ which relates the strain of a mercury-filled rubber tube by measuring change in electric resistance of metal due to its geometry change. Recently, mercury has been replaced with safer room-temperature liquid eutectic gallium alloys such as gallium-indium-tin (Galinstan). ${ }^{[2]}$ Besides polymer encapsulated strain sensors, ${ }^{[4]}$ these materials have also been proposed for a number of other applications including thermal interface materials, ${ }^{[5]}$ droplet-based micro-switches, ${ }^{[6]}$ microsyringes for cells, ${ }^{[7]}$ magneto-hydrodynamic pumps, ${ }^{[8]}$ and stretchable antennas. ${ }^{[9]}$ Because of the wide-range of potential applications, new routes of patterning liquid-metal structures aiming to replace the current manual syringe injection-molding process have been proposed. Examples of these methods include vacuum-induced patterning, ${ }^{[10]}$ contact printing, ${ }^{[11]}$ masked deposition, ${ }^{[12]}$ and oxide-stabilized 3D printing. ${ }^{[13]}$ However, fabrication of well-defined patterns with feature sizes below $50 \mu \mathrm{m}$ has not yet been achieved. The primary reasons limiting spatial resolution of these novel fabrication techniques are complex wetting dynamics and strong adhesion of gallium-based liquid metals to majority of materials resulting from rapid oxidation of the metal surface (e.g., formation of "oxide-sack" in Fig. 1a).

In this work, we use in-situ FIB-SEM imaging and manipulation to explore wetting and adhesive interactions of Galinstan and its oxide skin with a variety of materials to provide an insight into how these processes limit, but yet might be used to enhance, spatial resolution of current fabrication approaches. We observe the dynamics of oxide skin removal and regrowth after in-situ gallium and helium FIB abrasion (Fig. 1b \& c), as well as mechanical disruption using a tungsten manipulator (Fig. 1d). Furthermore, we study the nanoscale affinity of the native oxide skin, un-oxidized metal, as well as metal treated with hydrochloric acid (Fig. 1e) with manipulator needles modified with thin films of PTFE, PDMS, indium, and gallium. We demonstrate physical mechanisms limiting resolution of current pattern-transfer fabrication approaches and why these issues cannot be resolved through chemical removal of the oxide. Taking advantage of the oxide shell, we also demonstrate a novel "scoop-and-tap" procedure that allows individual sub-100 nm dots of Galinstan to be repeatedly transferred. 

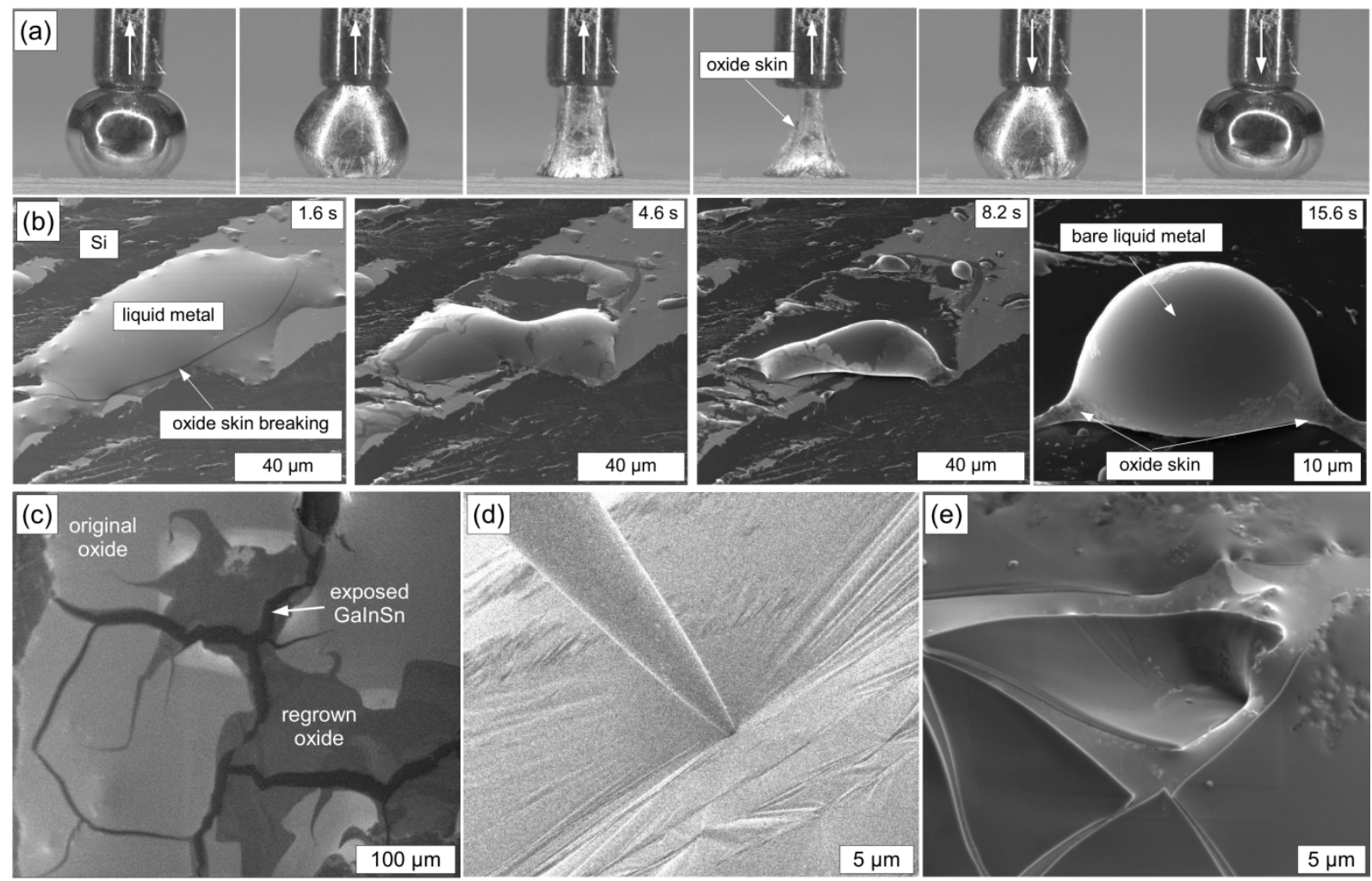

FIG. 1. (a) Dynamic contact angle measurements of Galinstan illustrating effects of oxide-skin formation, (b) dynamics of Galinstan de-wetting from silicon substrate due to ion irradiation induced oxide-skin removal, (c) regrowth of oxide with different thicknesses after several ion beam exposure cycles, (d) testing of elasticity and nanoscale affinity of oxide skin using surface modified-manipulator, and (e) modified liquid metal shell after hydrochloric acid treatment.

\section{References}

[1] D.-H. Kim, J. A. Rogers, Adv. Mat. (2008) 20.

[2] S. Cheng, Z. Wu, Lab Chip (2012) 12.

[3] R. Whitney, J. Physiology 1949, 109.

[4] R. K. Kramer, C. Majidi, R. Sahai, R. J. Wood, 2011 IEEE/RSJ, (2011).

[5] Q. Zhang, J. Liu, Nano Energy (2013).

[6] S. Wenjiang, R. T. Edwards, J. Y. Kim, J. MEMS (2006), 15.

[7] M. Knoblauch, J. M. Hibberd, J. C. Gray, A. J. van Bel, Nat. Biotechnol. (1999), 17.

[8] W. Irshad, D. Peroulis, Proc. Power MEMS, 2009.

[9] M. Kubo, et al., Adv. Mat. (2010), 22.

[10] B. L. Cumby, et al. App. Phys. Lett. (2012), 101; J. Park, et al. Nat. Comm. (2012) 3.

[II] S. H. Jeong, et al. Lab Chip (2012)12; A. Tabatabai, et al. Langmuir (2013);

[12] R. K. Kramer, C. Majidi, R. J. Wood, Adv. Funct. Mat. (2013).

[13] C. Ladd, J.-H. So, J. Muth, M. D. Dickey, Adv.Mat. (2013), 25.

Acknowledgements: KR: Funding from Fulton Schools of Engineering at ASU and NIST for use of FIB-SEM facilities. Part of the described worked was carried out at ASU's LeRoy Eyring Center for Solid State Science. Contact: konradr@asu.edu 